\title{
Building Lumped Models for Measured Passive mm-wave Components
}

\author{
Eero Sankila \\ University of Oulu \\ Oulu, Finland
}

\author{
Veeti Kiuru \\ University of Oulu \\ Oulu, Finland
}

\author{
Janne Aikio \\ University of Oulu \\ Oulu, Finland \\ janne.aikio@oulu.fi
}

\author{
Timo Rahkonen \\ University of Oulu \\ Oulu, Finland \\ timo.rahkonen@oulu.fi
}

\begin{abstract}
This paper presents a synthesis flow for building lumped circuit models of arbitrary complexity for mm-wave IC passive components, based on S-parameters obtained by measurements or electromagnetic (EM) field simulations. Lumped circuit models are needed in time-domain simulations, or to speed up the fine-tuning of passive circuit blocks, as iterating is much faster in circuit simulators than in EM simulator. Modeling algorithm is implemented in MATLAB, and the design flow has a few new features. The device model is given by Spice netlist, and its structure or complexity is not limited. Differential and common mode forms of admittance parameters are used to simplify solving the initial model component values that are then refined manually or by numerical optimization. The flow is illustrated by modeling a parallel $\mathrm{LC}$ resonator, whose response has been measured from 1 to $40 \mathrm{GHz}$.

Index Terms-lumped model, S-parameter, mixed-mode parameters
\end{abstract}

\section{INTRODUCTION}

Beyond $10 \mathrm{GHz}$, the distributed nature of on-chip wiring begins to make it necessary to use electro-magnetic simulations (EM) for generating models for passive components and routing. S-parameters obtained from EM-simulations can be used in harmonic balance simulations, but there are a few reasons why a lumped equivalent model is preferred [1]. First, the EM-simulated S-parameter models pose problems in time-domain periodic steady state analysis and initial transient analyses. Some simulators can build a transient model based on the impulse response of the S-parameter model, but that may suffer from too sparse frequency sampling - in the worst case, a passive circuit may appear active. Second, tweaking the component values in a lumped model gives you rapid understanding of what model parameters and geometric properties are causing which frequency effects, and may this way help to improve the design layout.

This paper presents a characterization flow of passive $\mathrm{mm}$ wave components and shows a few example cases, implemented in a $22 \mathrm{~nm}$ CMOS SOI process and measured directly on-chip from DC to $40 \mathrm{GHz}$ using probe station.

\section{Calibrating And De-Embedding the MEASUREMENTS}

The first step in the modeling measured structures is to deembed the effects of the fixture, IO pads and routing between pads and the device under test (DUT). This requires some

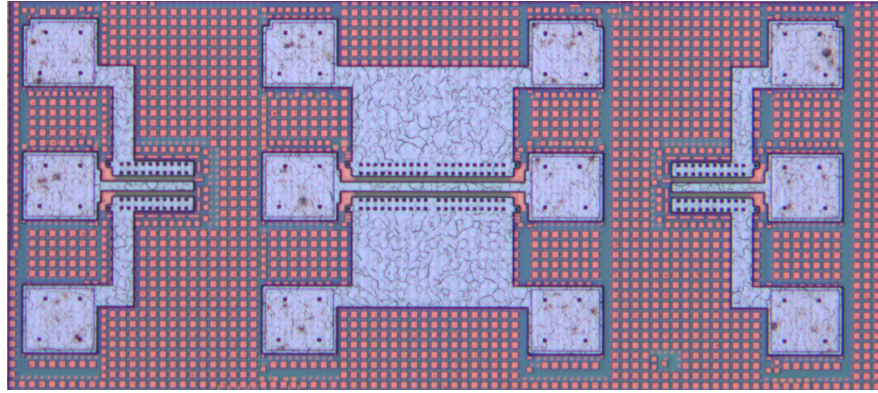

Fig. 1. Reflect and thru calibrating structures.

calibration structures, which usually starts by SOLT (shortopen-load-thru) calibration up to the probe tips using separate calibration substrates. Open-short-through [2], [3] calibration procedures are commonly used techniques. In this paper we used on-chip TRL (through-reflect-line) standards [4], which at the same time allows to extract a per-length model for the co-planar transmission line. After successful calibration, measured data of the actual DUT is de-embedded by peeling away the pad and leading wire. The reflect (left and right orientation) and thru structures are shown in Fig. 1.

\section{Choosing the Model Structure}

In PCB design the wiring structures are often modeled by building them of joined lines, T-sections, tapering blocks, etc. For a given IC metal stack such primitives do not usually exist, but the schematic structure of the model has to be built of basic circuit elements $(\mathrm{R}, \mathrm{L}, \mathrm{C})$ by inspection, and knowing that the model often needs to have more resonances that you want to model precisely. This is most easily explained by considering the 2-turn center-tapped coil in Figure 2. Model ports will be nodes 1, 2 and 3, and both 1-2 and 2-3 inductors are broken into two segments, each modeled by a normal pi model. Looking from layout, inductors L1 and L3 are close to each other, and will need a mutual coupling. The same applies for L2 and L4, while e.g. L1 and L2 are far from each other, and the coupling can be ignored. Apparently, capacitive coupling from node 3 to 4 and 5 is weak, but 1-3,23 and 4-5 couplings are strong. In practice, both $\mathrm{L}$ and $\mathrm{C}$ will be needing series losses, $\mathrm{L}$ often needs a skin effect model for better low-frequency match, and the series resistance of 

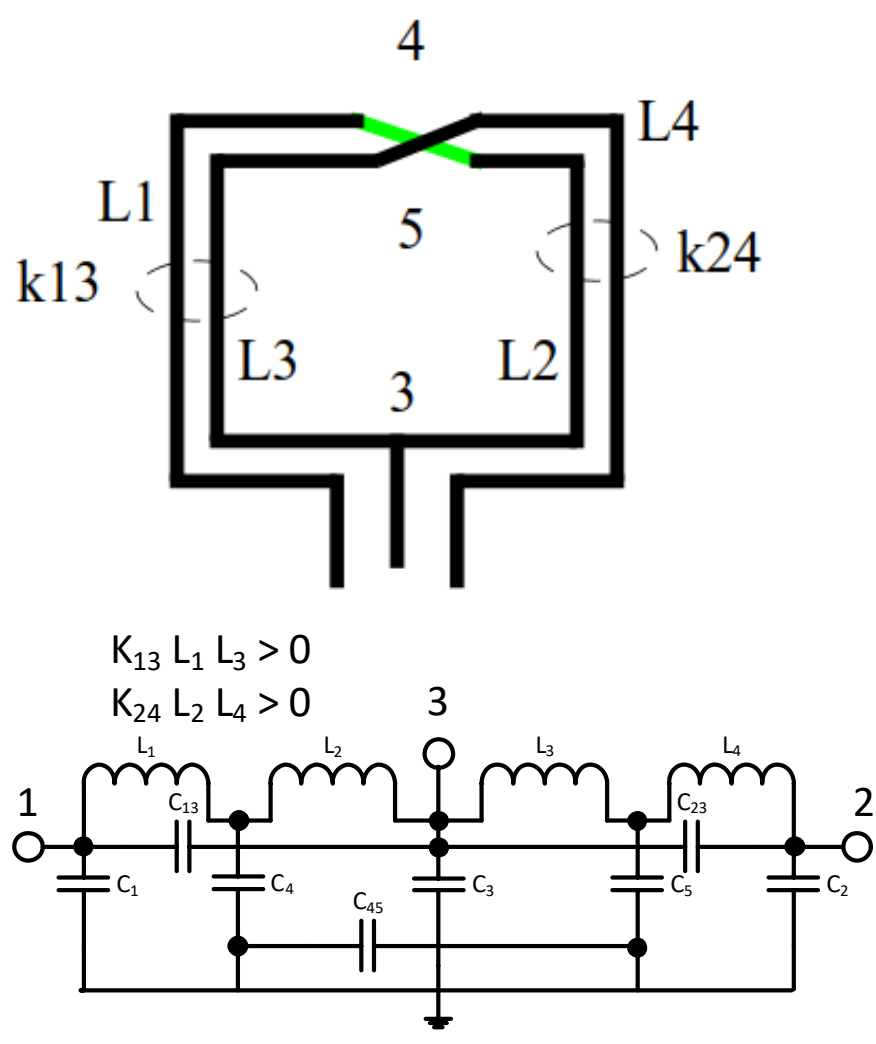

Fig. 2. Example 2-turn coil and corresponding model.

substrate capacitance (C1-C5) may have an additional shunt capacitance, too [6]. Employing the symmetry of the structure we can reduce the number of parameters, e.g. by setting $\mathrm{C} 1=\mathrm{C} 2=\mathrm{C} 3 / 2, \mathrm{~L} 1=\mathrm{L} 4$, etc.

\section{Modeling Flow}

Fitting the model parameters could be done in a circuit simulator, where $\mathrm{S}$ or Y parameters of the measured block and lumped model are easily compared. However, numerical optimization gets increasingly difficult with increasing number of variables, and we wanted to do as much analytical a priori calculations as possible (based mostly on the Y-parameter presentation, as it nicely reflects a pi model). Coding that in scripting languages like Ocean is possible, but a bit cumbersome, so we decided to use MATLAB, instead.

The problem with MATLAB is that it does not have a direct functionality to solve Y-parameters of a given circuit schematic. Luckily, we had an existing code that converts a spice netlist to a symbolic modified nodal analysis (MNA) matrix where we can still change all the component values. This is then numerically reduced to Z-parameters, which allows us to process models of any complexity, without tedious derivation of lengthy symbolic expressions.

The main novelty in this paper is that in addition to single-ended parameters, also differential and common-mode parameters are used [7]. The benefit of doing this for Yparameters as in [8] is that the common mode input admittance very nicely lumps all grounded components, and gives a quick estimate of the total capacitance towards the ground by fitting a capacitance response to low-frequency common-mode admittance.

Conversion from single-ended Y-parameters $Y_{s e}$ to mixed mode Y-parameters $Y_{m m}$ is calculated by matrix algebra as follows [8].

$$
Y_{m m}=M_{I} \cdot Y_{s e} \cdot M_{V}^{-1}
$$

Here $M_{I}$ and and $M_{V}$ represent the change of variables: single-ended port voltages and currents $V_{s e}, I_{s e}$ are changed to difference and average voltages and currents needed in the mixed-mode presentation.

$$
\begin{gathered}
V_{m m}=M_{V} \cdot V_{s e} \\
I_{m m}=M_{I} \cdot I_{s e}
\end{gathered}
$$

For example for two-port measurement data $Y_{m m}$ has the following contents

$$
Y_{m m}=\left(\begin{array}{cc}
Y_{\text {diff }} & Y_{\text {diffcm }} \\
Y_{\text {cmdiff }} & Y_{c m}
\end{array}\right)
$$

where $Y_{c m}$ and $Y_{\text {diff }}$ are the common-mode and differential admittances, and $Y_{\text {diff } c m}$ and $Y_{c m \text { diff }}$ describe the mode conversions due to asymmetries.

As an example, let us think a pi segment L1,C1,C4 between nodes 1 and 4 in Fig. 2 as a simple and separate two-port. Here $Y_{c m}$ is directly $j \omega(C 1+C 4)$, and $Y_{\text {diff }}=Y_{L 1}+$ the series connection of the capacitive branches. Hence, extracting the initial model parameters is quite simple. Extracting the losses, too, requires either choosing a proper frequency range or performing a least square error fit, and these are coded in the model function that contains the MNA matrix and solving of the Y-parameters: for initial values, mixed-mode parameters are used, while in later iterations, single-ended parameters are used to compare to the measured result.

Altogether, the flow (after de-embedding the pads) is the following: an arbitrary model is drawn as a schematic diagram; a function converts the spice netlist of the model to a symbolic MNA in MATLAB format, from which any parameter models can be numerically solved as functions of the model component values. The initial values for those are derived by the use of common mode and differential impedances, and then parameters of the measurement and the model are compared. Magnitude plot alone is not a sufficient measure. Often both real and imaginary parts are plotted, but phase is actually a very sensitive indication of new poles or zeros just outside the measured frequency range. Hence, as in Fig. 3, we plot magnitude and phase of the measurement and model on top of each other, and vector error (5) magnitude on $\mathrm{dB}$ scale as a FOM of the model fit. Residual error $E_{d B}$ clearly less than $-20 \mathrm{~dB}$ is aimed for over the entire frequency band.

$$
E_{d B}=20 \cdot \log _{10}\left|\frac{Y_{i j_{\text {meas }}}-Y_{i j_{\text {model }}}}{Y_{i j_{\text {meas }}}}\right|
$$



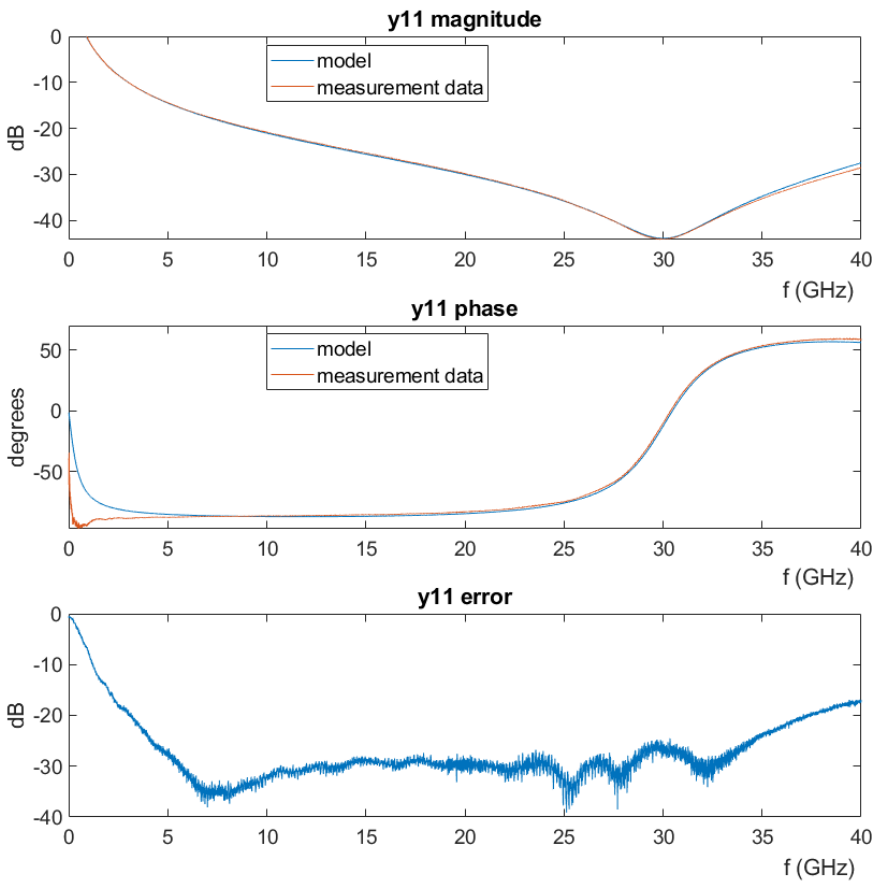

Fig. 3. Magnitude, phase and error plots of $Y_{11}$ of the parallel LC resonator. Model and measurement data compared.

Besides the vector error, it was found very valuable to swap between linear and logarithmic frequency scales, as log scale easily guides a lot of effort for modeling low-frequency effects, while in our case the performance around $20-40 \mathrm{GHz}$ is actually the most crucial. $-20 \mathrm{~dB}$ errors in Y-parameters did not guarantee as small errors in S-parameters, so it was found wise to check regularly plots and errors of S-parameters respectively while modeling. It was also found that inductor responses from $10 \mathrm{GHz}$ onwards could be modeled by single series RL models (actually two of those in series), but if simultaneous match is wanted at low frequencies, too, the skin effect needs to be modeled by additional RL branches to model the lower losses at low frequencies.

\section{MOdELING EXAMPLES}

As an example of the fitting procedure, the parallel resonator shown in Fig. 4 was modeled, consisting of 1-turn inductor and a tiny MOM capacitor between the legs of the inductor. The IO structure was peeled away, and the ports in the plot are now at the reference planes. The corresponding circuit model is shown in Fig.5. The capacitor model is a simple pi model with lossy parasitic capacitance to substrate (adding substrate time constant by additional caps in parallel to R6, R7 had no noticeable effect). The inductor was split into two segments, but as the halves are far from each other, no mutual coupling is needed, and capacitive coupling from the end to the center is also very weak. To keep the model simple, the skin effect was not taken into account here, resulting in a worse fit below $5 \mathrm{GHz}$.

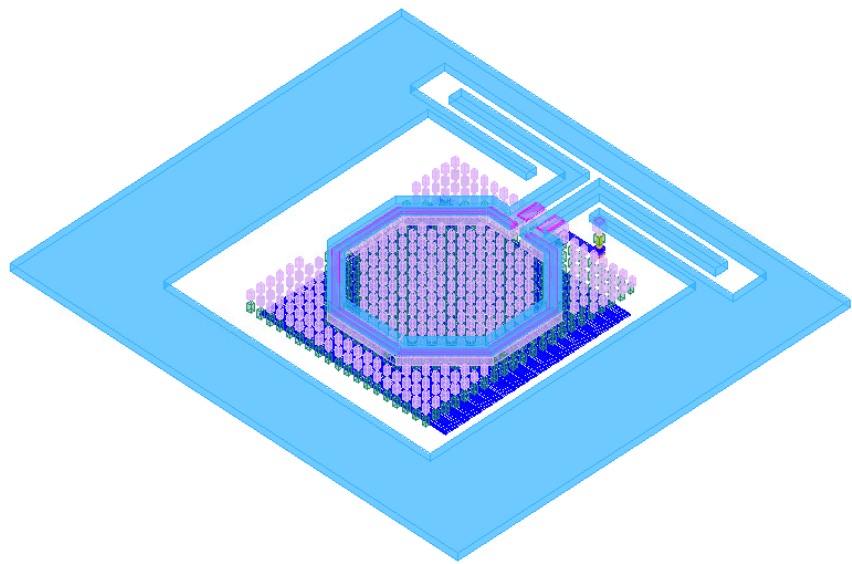

Fig. 4. 3D figure of the parallel LC resonator.

This parallel LC combination alone did not give a good phase at the highest frequencies, and looking at the $Y_{12}$ phase response suggested an emerging low-ohmic (series) resonance somewhere above $40 \mathrm{GHz}$. Brief look at the layout (Fig 4) reminded that the inductor legs from reference planes to the cap pins were rather long, and the model was extended by series inductors L3, L4in both ports to generate the needed high-frequency series L-C_- L-L series resonance. After this the measurement and model matched very well (see figure 6).

In the design kit used, and the measurement matched also quite well with the schematic models available in the kit. Surprisingly, the match with EM simulations was not that good. The fine geometries in the MOM capacitor, thin lines in the inductor shield, and fill metal generated lot of layout geometry, and the layout needed to be simplified a lot to be able to simulate it. In the first simulations, the capacitor was replaced with its schematic model, and inductor shield lines were partially merged to reduce the number of edges. Still especially $S_{11}$ and $S_{22}$ matched poorly with the measurements, and the model parameters were varied to see what would cause such behavior. Simulation sweeps with the lumped model suggested that capacitors $\mathrm{C} 1-\mathrm{C} 3$ could be the cause, so the original shield geometry was returned. A new and now much longer simulation showed a better match for $S_{12}$ resonance, but still gave overall wrong shape for low-frequency $S_{11}$ and $S_{22}$. Reasons for this discrepancy are still under study.

\section{Discussion}

Building device models from probe measurements is a rather well matured art, and some commercial modeling services exist, too. What is believed to be new in our approach is the following: First, we can automatically change any selected group of single-ended ports to differential and common-mode ports, if needed and this actually helps to extract the total grounded admittance and to find an initial guess for the portto-port couplings. Second, if the built schematic model is physically well motivated, it gives the designer a way to quickly simulate what to do to improve the design. Hence, we are not limiting to modeling already implemented circuits, 




Fig. 5. Model for the parallel LC resonator.

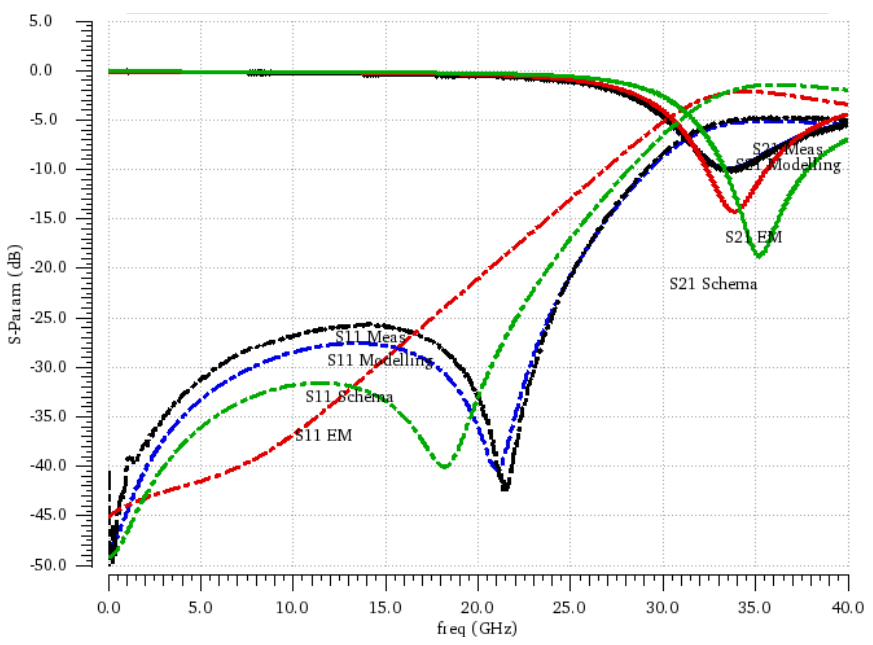

Fig. 6. Comparison of S-parameters of the pdk schematic model, measurement, fitted model, and EM model.

but plan to use this as a design tool, too: the circuit design can be fine-tuned with fast simulations at the circuit level, and the found solution can be reflected to a layout dimensioning change, and then verified by EM simulations. As the EM simulations can take hours, this iteration speed-up is more than welcome.
In summary, the modeling procedure is not restricted to model library building or generating models suited for timedomain simulations, but we propose to use it also as a guiding tool in the layout iteration phase, to minimize the iterations spent in the EM simulations. Future work includes improving the numerical optimization after the initial model values have been calculated.

\section{ACKNOWLEDGMENT}

This research has been financially supported by Academy of Finland 6Genesis Flagship (grant 318927) and Nokia Corporation Ltd. Global Foundries is acknowledged for silicon fabrication.

\section{REFERENCES}

[1] M. S. Sarto, A. Scarlatti and C. L. Holloway, "On the use of fitting models for the time-domain analysis of problems with frequency-dependent parameters," 2001 IEEE EMC International Symposium. Symposium Record. International Symposium on Electromagnetic Compatibility (Cat. No.01CH37161), Montreal, Que., 2001, pp. 588-593 vol.1. doi: 10.1109/ISEMC.2001.950710

[2] H. Cho and D. E. Burk, "A three-step method for the de-embedding of high-frequency S-parameter measurements," in IEEE Transactions on Electron Devices, vol. 38, no. 6, pp. 1371-1375, Jun 1991. doi: $10.1109 / 16.81628$

[3] E. P. Vandamme, D. M. M. P. Schreurs and G. Van Dinther, "Improved three-step de-embedding method to accurately account for the influence of pad parasitics in silicon on-wafer RF test-structures," in IEEE Transactions on Electron Devices, vol. 48, no. 4, pp. 737-742, Apr 2001. doi: $10.1109 / 16.915712$

[4] G. F. Engen and C. A. Hoer, "Thru-Reflect-Line: An Improved Technique for Calibrating the Dual Six-Port Automatic Network Analyzer,' in IEEE Transactions on Microwave Theory and Techniques, vol. 27, no. 12, pp. 987-993, Dec 1979. doi: 10.1109/TMTT.1979.1129778

[5] R. O. Topaloglu, J. S. Goo, A. L. S. Loke, M. M. Oshima and S. W. Sim, "SIS wide-band model extraction methodology for SOI on-chip inductor," 2010 International Conference on Microelectronic Test Structures (ICMTS), Hiroshima, 2010, pp. 90-93. doi: 10.1109/ICMTS.2010.5466850

[6] A. C. Watson, D. Melendy, P. Francis, Kyuwoon Hwang and A. Weisshaar, "A comprehensive compact-modeling methodology for spiral inductors in silicon-based RFICs," in IEEE Transactions on Microwave Theory and Techniques, vol. 52, no. 3, pp. 849-857, March 2004. doi: 10.1109/TMTT.2004.823594

[7] D. E. Bockelman and W. R. Eisenstadt, "Pure-mode network analyzer for on-wafer measurements of mixed-mode S-parameters of differential circuits," in IEEE Transactions on Microwave Theory and Techniques, vol. 45, no. 7, pp. 1071-1077, Jul 1997. doi: 10.1109/22.598443

[8] T. Rahkonen and J. Kortekangas, "Mixed-mode parameter analysis of fully differential circuits," 2004 IEEE International Symposium on Circuits and Systems (IEEE Cat. No.04CH37512), 2004, pp. I-269-I272 Vol.1. doi: 10.1109/ISCAS.2004.1328183 